\title{
Universiteit
}

Leiden

The Netherlands

\section{Quantum-to-classical crossover of mesoscopic conductance} fluctuations

Beenakker, C.W.J.; Tworzydlo, J.; Tajic, A.

\section{Citation}

Beenakker, C. W. J., Tworzydlo, J., \& Tajic, A. (2004). Quantum-to-classical crossover of mesoscopic conductance fluctuations. Retrieved from https://hdl.handle.net/1887/1291

Version: $\quad$ Not Applicable (or Unknown)

License: $\quad$ Leiden University Non-exclusive license

Downloaded from: https://hdl.handle.net/1887/1291

Note: To cite this publication please use the final published version (if applicable). 


\title{
Quantum-to-classical crossover of mesoscopic conductance fluctuations
}

\author{
J Tworzydlo ${ }^{1}$ ' A Tajk ' and C W J Beenakke1 ${ }^{1}$ \\ 'Instuluut Lorcht Unucisulet Luden PO Box 95062300 RA Letden The Netherlands \\ 'Instutute of theoictical Phisics Warsau Unticisti Ho a 6900681 Warsan Poland \\ (Recerved 12 Norembcr 2003 icrised munuscipl iccered 15 Jununy 2004 published 27 Apul 2004)
}

\begin{abstract}
We calculite the system st/c oret wivelength $(M)$ dependence of sample to sample conduct ince fluctua toons using the open hiched iot itor to model chrotic scitteing in a ballistic quantum dot coupled by two $N$ mode point contacts to election reservous Both i fully quantum mechanical and a semiclassical calculation ate piesented and found to be in good igiecment The mean squated conduct mce fluctuations ie ich the universal quantum limit of iandom matıx theory for smull systems For latge systems they inciease $r M^{\prime}$ dt fixed mean dwell tume $\tau_{D} \leq M / N$ The universal quantum fluctuations dommate over the nonuniversal classic al fuctuations if $N<\sqrt{M}$ When expressed as a 1 atio of time scales the quantum to classical crossovel is gor eined by the ratio of Ehienfest time and ergodic time
\end{abstract}

DOI 10 1103/PhysRevB 69165318

PACS number(s) $7323-$ b $7363 \mathrm{~K}, 0545 \mathrm{Mt} 0545 \mathrm{Pq}$

\section{INTRODUCTION}

Sample to sample fluctuations of the conductance of dis ordered systems have a universal regime 11 which they are independent of the mean conductance The requirement for these universal conductance fluctuations ${ }^{\text {" }}$ is that the sample size should be small compared to the localization length The mean conductance is then much larger than the conductance quantum $e^{7} / h$

The same condition applies to the univeisality of conduc tance fluctuations in ballistic chaotic quantum dots ${ }^{34}$ al though theie is no localization in these systems Random matrix theory (RMT) has the unversal limit

$$
\lim _{N \rightarrow r} \operatorname{var} G=\frac{1}{8}
$$

for the valiance of the conductance $G$ in units of $e^{3} / h$ Here $N$ is the number of modes tiansmitted through each of the two ballistic point contacts that connect the quantum dot to electron reservors Since the mean conductance $\langle G\rangle=N / 2$ the condition for univeisality remans that the mean conduc tance should be lage compared to the conductance quantum

In the present paper we will show that there is actually an upper limit on $N$ beyond which RMT bieaks down in a quantum dot and the unis ersality of the conductance fluctua tions is lost Since the width $W$ of a point contact should be small compared to the size $L$ of the quantum dot in order to have chaotic scatteing d tirval requilement is $N \ll M$ where $M$ is the number of transverse modes in a cioss section of the quantum dot (In two dimensions, $N \simeq W / \lambda_{\Gamma}$ and $M$ $\simeq L / \lambda_{\Gamma}$ with $\lambda_{\text {I }}$ the $\Gamma$ eimi wavelength ) By consideing the quantum to classical crossover we antive at the more stim gent requilement

$$
1 \ll N \ll \sqrt{M} c^{\lambda} \quad \rho
$$

with $\lambda$ the Lyapunos exponcnt and $\tau_{\text {el }}$ the elgodic time of the classical chaotic dynamics The tequitement is more stingent than $N \ll M$ because ryprally $\lambda^{1}$ and $\tau_{\mathrm{c}}$ a are both equal to the time of flight $\tau_{0}$ acioss the system so the exponential factor in Eq (1 2) is not far from unity

Expressed in teims of time scales the upper limit in Eq ( 12 ) says that $\tau_{\mathrm{el}}$, should be larger than the Ehrentest tıme

$$
\tau_{F}=\max \left[0 \lambda \quad{ }^{1} \ln \frac{N^{\prime}}{M}\right]
$$

The condition $\tau_{\text {eIs }}>\tau_{L}$ which we find for the universality of conductance fluctuations is much more stingent than the condition $\tau_{D}>\tau_{F}$ for the validity of RMT found in other contexts $^{3-1}{ }^{\prime}$ Here $\tau_{D} \approx(M / N) \tau_{0}$ is the mean dwell tume $n$ the quantum dot which is $\geqslant \tau_{\text {ug }}$ in any chaotic system

The outhne of this paper is as follows In Sec II we describe the quantum-mechancal model that we use to cal culate val $G$ numerically which is the same stroboscopic model used in previous investigations of the Ehrenfest time ${ }^{91114}$ The data ate interpieted semiclassically in Sec III leading to the ciossovei criterion (1 2) We conclude in $\mathrm{Sec}$ IV

\section{STROBOSCOPIC MODCL}

The physical system we have in mind is a ballistic (clean) quantum dot in a two dimensional election gas connected by two ballistic leads to election reseivoris While the phase space of this system is four dimensional it can be teduced to two dimensions on a Poncale sufface of section ${ }^{1716}$ The open kicked rotator $91114>017-19$ is a stioboscopic model with a two dimensional phase space We summarize how this model is constucted following Ref $1 \mathrm{I}$

One stat from the closed system (without the leads) The kicked sotator describes a particle moring along a cucle kıcked perodically at time intervals $\tau_{0}$ We set to unity the stroboscopk time $\tau_{0}$ and the Plank constant $\hbar$ The stiobo scoptc time evolution of a wave function is given by the Floquet operator $\mathcal{F}$ which can be represented by an $M$ $\times M$ untary symmetıc matuid The elen mteger $M$ defines the effective Planch constant $h_{41}-1 / M$ In the disciete cool dinate representation $(1=m / M \quad m=01 \quad M-1)$ the matux elements of $\mathcal{F}$ ale given by 


$$
\left.\mathcal{F}_{1 \mathrm{~m} m}=M^{1 / 2} e^{i \tau / 4} e^{i 2 \pi M S(\imath} \backslash\right),
$$

where $S$ is the map generating tunction,

$$
S\left(x^{\prime} x\right)=\frac{1}{2}\left(x^{\prime}-x\right)^{2}-\left(K / 8 \pi^{2}\right)\left(\cos 2 \pi x^{\prime}+\cos 2 \pi x\right)
$$

and $K$ is the kicking stiength

The elgenvalues $\exp \left(-l \varepsilon_{m}\right)$ of $\mathcal{F}$ define the quasieneigies $\varepsilon_{n} \in(02 \pi)$ The mean spacing $2 \pi / M$ of the quasienergies plays the cole of the mean level spacing $\delta$ in the quantum dot

To model a pan of $N$ mode ballistic leads, we impose open boundary conditions in a subspace of Hilbert space represented by the indices $m_{n}^{(\alpha)}$ The subscipt $n$ $=1,2, \quad N$ labels the modes and the supersciupt $\alpha=1,2$ la bels the leads A $2 N \times M$ projection matix $P$ describes the coupling to the ballistic leads Its elements ate

$$
P_{n, 1}= \begin{cases}1 & \text { if } m=n \in\left\{m_{n}^{(\alpha)}\right\} \\ 0 & \text { otherwise }\end{cases}
$$

The mean dwell time is $\tau_{D}=M / 2 N$ (in units of $\tau_{0}$ )

The matrices $P$ and $\mathcal{F}$ together determine the quasienergy dependent scattening matı $1 \mathrm{x}$

$$
S(\varepsilon)=P\left[e^{-\imath \varepsilon}-\mathcal{F}\left(1-P^{\mathrm{T}} P\right)\right]{ }^{1} \mathcal{F} P^{\mathrm{T}}
$$

The symmetry of $\mathcal{F}$ ensures that $S$ is also symmetic, as $1 \mathrm{t}$ should be $i n$ the presence of time-reversal symmetry By glouping together the $N$ indices belonging to the same lead the $2 N \times 2 N$ matnx $S$ can be decomposed into foul sub blocks containing the $N \times N$ transmission and reffection ma tilces,

$$
S=\left(\begin{array}{cc}
1 & t \\
t^{\prime} & ,
\end{array}\right)
$$

The conductance $G$ (in units of $e^{2} / h$ ) follows fiom the Lan dauer formula

$$
G=T_{1} \quad t t^{\dagger}
$$

The open quantum kicked 1otator has a classical limit described by a map on the tor us $\{\lambda p \mid$ modulo $\}$ The class1cal phase space, including the leads, is shown in Fig 1 The map relates $x, p$ at time $k$ to $\imath^{\prime}, p^{\prime}$ at time $k+1$

$$
p^{\prime}=\frac{\partial}{\partial x^{\prime}} S\left(x^{\prime}, x\right) \quad p=-\frac{\partial}{\partial x} S\left(x^{\prime} x\right)
$$

The classical mechanics becomes fully chaotic for $K \geq 7$, with Lyapunov exponent $\lambda \approx \ln (K / 2)$ Fol smalles $K$ the phase space is mixed contanng both iegions of chaotic and of regula1 motion We will restrict ourselves to the fully chaotic legime in this paper

\section{NUMI RICAI RCSULIS}

To calculate the conductance (26) we need to invert the $M \times M$ matix between square biackets in Eq (24) We do this numerically using an iterative procedure ${ }^{11}$ The iteration

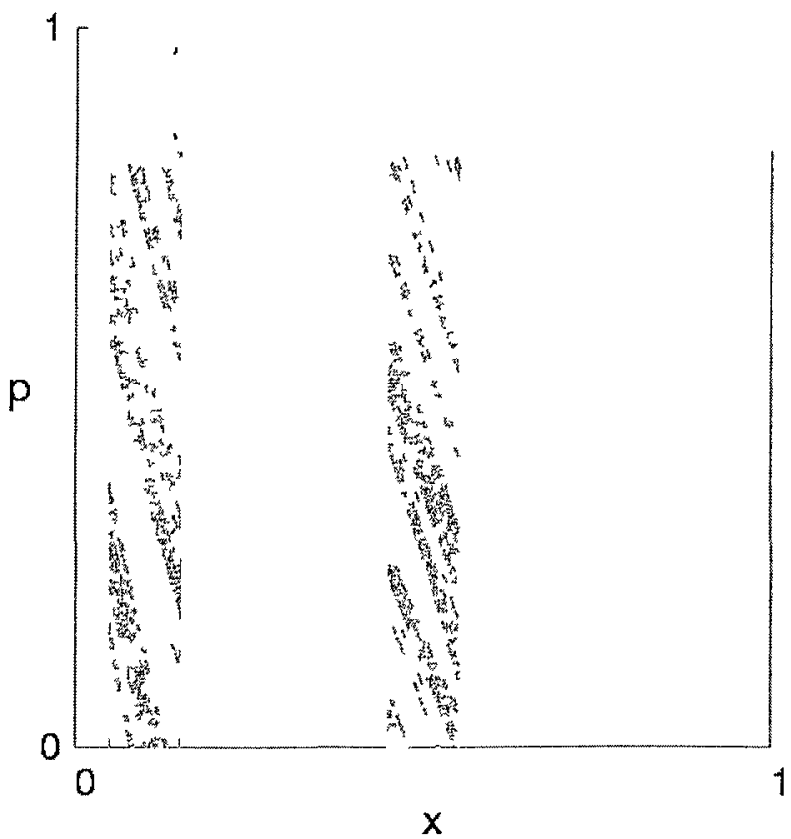

FIG I Classical phise space of the open kicked rotator The dashed lines indicate the two leads (shown for the case $\tau_{D}$ 5) Inside each lead we plot the initial and final coordinates of tiajec tones which ate tunsmitted fiom the lelt to the inght lead after at most thee iterations (with $K=75$ ) The points cluster along nar low ti nnsmission bands

can be done efficiently using the fast Founter-tiansform algo isthm to calculate the application of $\mathcal{F}$ to a vector The time required to calculate $S$ scales as $M^{2} \ln M$ which for laige $M$ is quicke than the $M^{\prime}$ scaling of a diect inversion The memory requnements scale as $M$, because we need not store the tull scattering matux to obtan the conductance

We distinguish two types of mesoscopic fluctuations in the conductance The first type appears upon valying the quasienergy $\varepsilon$ for a given scattering matix $S(\varepsilon)$ Since these fluctuations have no classical analog [the classical map (27) being $\varepsilon$ independent], we refer to them as quantum fluctua tions The second type appeas upon valying the position of the leads so these involve valiation of the scatteing matix at fixed $\varepsilon$ We refer to them as sample to sample fluctua tions They have both a quantum mechancal component and a classical analog One could intioduce a thisd type of fluc tuations involving both variation of $\varepsilon$ and of the lead posi tions We have found (as expected) that these are statistically equivalent to the sample to-sample fluctuations at fixed $\varepsilon$ so we need not distingursh between fluctuations of type 2 and 3

We have calculated the vartance var $G=\left\langle G^{2}\right\rangle-\langle G\rangle^{2}$ of the conductance either by valying $\varepsilon$ at fixed lead positions (quantum fluctuations) or by valying both $\varepsilon$ and lead posi tions (sample to sample fluctuations) Since the quantum in teiterence patten is completely ditferent only tor energy valuations of order of the Thouless energy $1 / \tau_{D}$ we choose a number $\tau_{D}$ of equally spaced values of $\varepsilon$ in the interval $(02 \pi)$ We take ten different lead positions, iandomly lo cated at the 1 axis in Fig 1 To investigate the quantum to classical crossover we change $h_{\mathrm{ctf}}=l / M$ while keeping the 


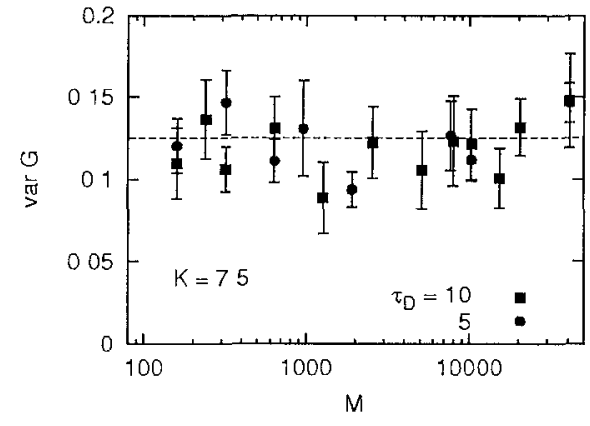

FIG. 2. Variance of the conductance fluctuations obtaned numerically by varying $\varepsilon$ with fixed lead positions. Error bars indicate the scatter of values obtained for different lead positions. Results are shown as a function of $1 / h_{\mathrm{eft}}=M$, for two values of the dwell ume $\tau_{D}=M / 2 N$. The dashed line is the RMT prediction var $G$ $=\frac{1}{8}$.

dwell time $\tau_{D}=M / 2 N$ constant. The results are plotted in Figs. 2 and 3.

\section{INTERPRETATION}

We interpret the numerical data by assuming that the variance of the conductance is the sum of two contributions: a universal quantum-mechanical contribution $\mathcal{V}_{\mathrm{RMT}}$ given by random-matrix theory and a nonuniversal quasiclassical contribution $\mathcal{V}_{c l}$ determined by sample-to-sample fluctuations in the classical transmission probabilities.

The RMT contribution equals 3.4

$$
\mathcal{V}_{\mathrm{RMT}}=\frac{1}{8}
$$

in the presence of time-reversal symmetry. The classical contribution is calculated from the classical map (2.7), by determining the probability $P_{1-2}$ of a particle injected randomly through lead 1 to escape via lead 2 . Since the conductance is given semiclassically by $G_{\mathrm{cl}}=N P_{1-2}$, we obtain

$$
V_{\mathrm{cl}}=N^{2} \text { var } P_{1 \rightarrow 2}
$$

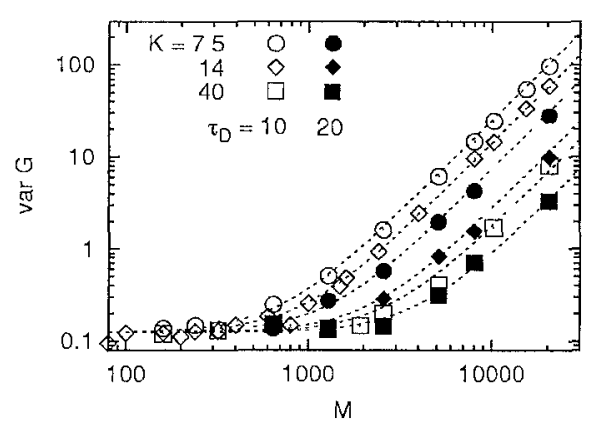

FIG 3 Same as Fig. 2, but now for an ensemble wn which the lead positions and the culasienergy are both varied The dashed lines are the sum of the RMT value (4.1) and the classical result (4.2). Results are shown for thice values of the kicking sticngth $K$ Open symbols are for the dwell tume $\tau_{D}=10$ and closed ones for $\tau_{D}$ $=20$

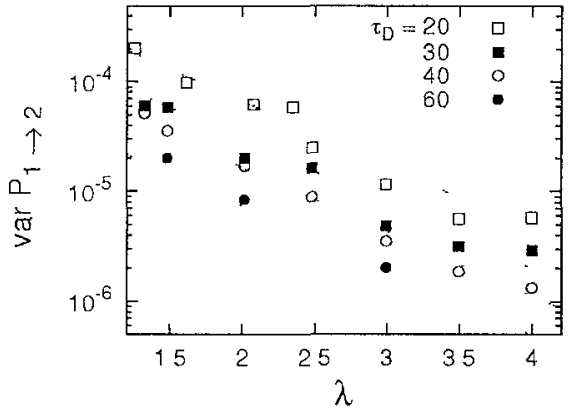

FIG. 4. Varrance of the classical fluctuations of the transmission probability $P_{1-2}$ upon changes of lead positions, calculated numerically from the map (2.7). The data are shown for four values of the dwell tume $\tau_{D}$, as a function of the Lyapunov exponent $\lambda$ $=\ln (K / 2)$. The dotted lines are the analytical prediction (4.3), with fit parameters $c=1.6$ and $\tau_{\text {cig }}=0.68$ (the same for all data sets).

We plot var $G=V_{\text {RMT }}+V_{\text {Cl }}$ in Fig. 3 (dashed curves), for comparison with the results of our full quantum-mechanical calculation. The agreement is excellent.

We now would like to investigate what ratio of time scales governs the crossover from quantum to classical fluctuations.

To estimate the magnitude of the sample-to-sample fluctuations in the classical transmission probability, we use results from Ref. 6 . There it was found that the starting points (and end points) of transmitted trajectories are not homogeneously distributed in phase space. Instead, they cluster together in nearly parallel, narrow bands. These transmission bands are clearly visible in Fig. 1. The largest band has an area $A_{\text {max }}=A_{0} e^{-\lambda \tau_{\text {eIg }}}$ determined by the ergodic time $\tau_{\text {elg }}$. This is the time required for a trajectory to explore the whole accessible phase space. The values of $\tau_{\mathrm{e} 1 \mathrm{~g}}$ and $A_{0}$ depend on the degree of collimation of the beam of trajectories injected into the system. ${ }^{6}$ For our model, without collimation, one has $\tau_{\text {cIg }}$ of order unity (one stroboscopic period) and $A_{0}$ $\simeq(N / M)^{2}$. The typical transmission band has an area $A_{0} e^{\left.-\lambda \tau_{l}\right)}$ which is exponentially smaller than $A_{\max }$ (since $\tau_{D}=M / 2 N \gg \tau_{\mathrm{erg}}$ ).

As the position of the lead is moved around, transmission bands move into and out of the lead. The resulting fluctuations in the transmission probability $P_{1 \rightarrow 2}$ are dominated by the largest band. Since there is an exponentially large number $e^{\lambda \tau_{D}}$ of typical bands, their fluctuations average out. The total area in phase space of the lead is $A_{\text {lead }}=N / M$, so we estimate the mean-squared fluctuations in $P_{1-2}$ at

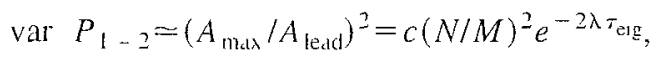

with $c$ and $\tau_{\text {erg }}$ of order unity. We have tested this functional dependence numerically for the map (2.7), and find a reasonable agreement (see Fig. 4). Both the exponential dependence on $\lambda$ and the quadratic dependence on $\tau_{D}=M / 2 N$ are consistent with the data. We find $\tau_{\text {elg }}=0.68$ of order unity, as expected.

Equations (4.2) and (4.3) mply

$$
\text { var } G=\frac{1}{8}+c\left(N^{+} / M^{2}\right) e^{-2 \lambda \tau_{\mathrm{etg}}}
$$




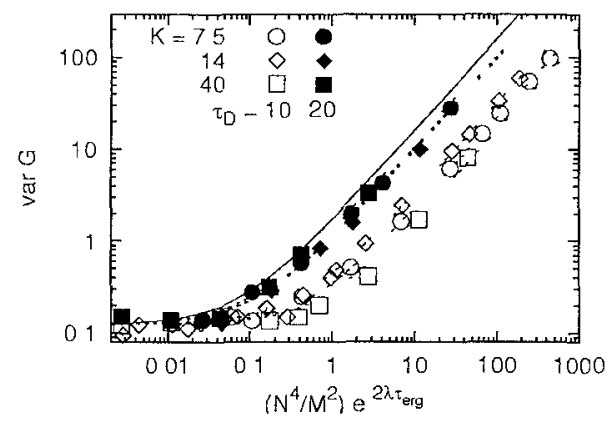

FIG 5 Same data as in Fig 3 rescaled to show the approach to a single limiting curve in the latge dwcll tume limit The solid line is calculated fiom Eq (44), with the same parameters $c=16, \tau_{\text {erg }}$ $=068$ as in Fig 4

In Fig 5 we plot the same data as 10 Fig 3, but now as a function of $\left(N^{4} / M^{2}\right) e^{-2 \lambda \tau_{\mathrm{e} 1}}$ We see that the functional dependence (44) is approached tor laige dwell times

The quantum fluctuations of RMT dommate over the classical fluctuations if $N^{2}$ val $P_{1-2} \ll 1$ Using the estimate (43), this amounts to the condition

$$
\tau_{\text {elg }}>\max \left[0, \lambda^{-1} \ln \left(N^{2} / M\right)\right] \equiv \tau_{F}
$$

that the eigodic time exceeds the Ehrentest time Notice that condition (45) is always satisfied if $N^{2}<M \equiv 1 / h_{\text {eff }}$ This agiees with the findings of Ref 6 that the breakdown of RMT stat ts when $N \geq \sqrt{M}$

\section{CONCLUSIONS}

In summaty, we have presented both a fully quantummechanical and a semiclassical calculation of the quantumto-classical crossover from universal to nonuniversal conductance fluctuations The two calculations are in very good agreement, without any adjustable par ameter (compare data points with curves in Fig 3) We have also given an analyt1cal approximation to the numerical data, which allows us to determme the parametic dependence of the crossover

We have found that universality of the conductance fluctuations requires the ergodic time $\tau_{\text {elg }}$ to be larger than the Ehienfest time $\tau_{D}$ This condition is much more stringent than the condition that the dwell time $\tau_{D}$ should be laiget than $\tau_{E}$, found previously for universality of the shot norse in a quantum dot ${ }^{61011}$ The universality of the excitation gap in a quantum dot connected to a supeiconductor is also goven 1 ed by the 1 atio $\tau_{D} / \tau_{E}$ ather than $\tau_{\mathrm{erg}} / \tau_{L},{ }^{57-9}$ as $1 \mathrm{~s}$ the unversality of the weak-localization effect ${ }^{1213}$ These two properties have in common that they represent ensemble averages, 1 ather than sample-to-sample fluctuations

We propose that what we have found here for the conduc- tance is generic for other transport properties That the breakdown of RMT with increasing $\tau_{L}$ occuss when $\tau_{L}$ $>\tau_{D}$ for ensemble averages and when $\tau_{L}>\tau_{\text {erg }}$ for the fluctuations This has immediate experimental consequences, because it is much easier to violate the condition $\tau_{D}>\tau_{\text {erg }}$ than the condition $\tau_{E}>\tau_{D}$

To test this pioposal, an obvious next step would be to deteimine the idtro of time scales that goven the bieakdown of univessality of the fluctuations in the superconducting excitation gap The numeical data in Refs 14 and 21 were interpieted in terms of the ratio $\tau_{L} / \tau_{D}$, but an alteinative desciption in teims of the 1 atio $\tau_{E} / \tau_{\text {erg }}$ was not considered

One final remark about the distinction between classical and quantum fluctuations (explaned in Sec III) is as follows It is possible to suppiess the classical fluctuations entwely, by varying only the quasieneigy at fixed lead positrons In that case we would expect the breakdown of universality to be governed by $\tau_{D} / \tau_{L}$ instead of $\tau_{\mathrm{erg}} / \tau_{E}$ Our numeical data ( $\left.F_{1 g} 2\right)$ do not show any systematic deviation fiom RMT, probably because we could not leach sufficiently laige systems in ou1 simulation

\section{Note added}

Our final remark above has been criticized by Tacquod and Sukhor ukov ${ }^{22}$ They argue that the numerical data of $F_{1} g$ 2 (and simila data of then own) do not show any systematic deviation from RMT because quantum fluctuations iemain unversal if $\tau_{F}>\tau_{D}$ Then argument relies on the assumption that the effective RMT of Ref 6 holds not only for the classical fluctuations (as we assume hete), but also for the quantum fluctuations The effective RMT says that quantum fluctuations are due to a number $N_{\mathrm{cff}} \approx N e^{\left.-\tau_{l} / \tau_{l}\right)}$ of transmission channels with a RMT distibution Universality of the quantum fuctuations is then guaranteed even if $N_{\text {eff }} \ll N$, as long as $N_{\text {eff }}$ is still large compared to unity

This line of leasoning, if puisued further, contradicts the established theor $\mathrm{y}^{12}$ of the $\tau_{E}$ dependence of weak localization RMT says that the weak-localization cortection $\delta G$ $=-\frac{1}{4}$ is independent of the number of channels ${ }^{34}$ Validity of the effective RMT at the quantum level would therefore imply that weak localization temans univessal if $\tau_{\Gamma}>\tau_{D}$, as long as $N e^{-\tau_{l} / T_{D} \gg 1}$ This contradicts the result $\delta G$ $=\frac{1}{4} e^{-\tau_{1} / \tau_{i}}$ of Rets 12 and 13

\section{ACKNOWLEDGMENTS}

Th1s work was suppoited by the Dutch Scrence Foundauon NWO/FOM J T acknowledges the financial suppoit provided through the European Community's Human Potentad Plogiam under Contlact No HPRN-CT-2000-00144, Nanoscale Dynamics

\footnotetext{
${ }^{1}$ B L Altshuler Pis ma Zh Elsp Tcor FiL 41530 (1985) [3ETP Lett 41648 (1985)]

${ }^{2} \mathrm{PA}$ Lee and A D Stone Phys Rer Lett 55, 1622 (1985)

${ }^{3} \mathrm{H}$ U Baranger and PA Mello Phys Rev Lett 73, 142 (1994)
}

\footnotetext{
${ }^{4} \mathrm{R}$ A Jalabeil I-L Pichard and CW I Bcenakher Curophys Letl 27255 (199-t)

'M G Vavilov and A I Lakin, Phys Rev B 67115335 (2003)

${ }^{6} \mathrm{PG}$ Silvestiov, M C Goorden, and C WJ Beenakke Phys Rev
} 
B 67, 241301 (2003)

${ }^{7}$ A Loddeı and Yu V Nazarov, Phys Rev B 58, 5783 (1998)

${ }^{\circ}$ PG Silvesiov M C Goorden, and C W J Beenakkeı, Phys Rev Letl 90, 116801 (2003)

${ }^{9} \mathrm{Ph}$ Jacquod, H Schomerus, and CWJ Beenakket, Phys Rev Lett 90, 207004 (2003)

${ }^{10} \mathrm{O}$ Agam, 1 Aleıneı, and A Laıkn, Phys Rev Lett 85, 3153 (2000)

"J Tworzydto, A Tafic, H Schomerus, and CWJ Beenakkel, Phys Rev B 68, 115313 (2003)

${ }^{12}$ I L Alemel and A I Laıkın, Phys Rev B 54, 14423 (1996)

${ }^{13}$ I Adagideli. Phys Rev B 68. 233308 (2003)

${ }^{14} \mathrm{M} \mathrm{C}$ Gootden, Ph Jacquod, and CW J Beenakket, Phys Rev B 68, 220501 (2003)
${ }^{1}$ E B Bogomolny Nonlineanty 5805 (1992)

${ }^{16}$ R E Prange. Phys Rer Lett 90, 070401 (2003)

${ }^{17} \mathrm{~F}$ Borgonovi I Guarne1, and DL Shepelyansky. Phys Rev A 43, 4517 (1991)

${ }^{18} \mathrm{~F}$ Borgonov and I Guarne11, J Phys A 25, 3239 (1992)

${ }^{19} \mathrm{Y} V$ Fyodorov and $\mathrm{H}-\mathrm{J}$ Sommels. Pis ma Zh Eksp Teor $\mathrm{F}_{1 /}$ 72, 605 (2000) [JETP Lett 72, 422 (2000)]

${ }^{20} \mathrm{~A}$ Ossipor $\mathrm{T}$ Kottos, and $\mathrm{T}$ Gersel, Europhys Lett 62.719 (2003)

${ }^{21}$ A Kolmanyos, Z Kautmann, C J Lambelt, and J Csertı, Phys Rev B 67, 172506 (2003)

${ }^{22} \mathrm{Ph}$ Jacquod and EV Sukhorukov, Phys Rev Lett 92, 116801 (2004) 